\title{
Butyric Acid in Saliva of Chronic Periodontitis Patients Induces Transcription of the EBV Lytic Switch Activator BZLF1: A Pilot Study
}

\author{
RYO KOIKE ${ }^{1,2}$, KEIKO NODOMI $^{2}$, NORIHISA WATANABE ${ }^{2}$, YORIMASA OGATA ${ }^{3}$, OSAMU TAKEICHI ${ }^{4}$, \\ MASAMI TAKEI ${ }^{5}$, TADAYOSHI KANEKO ${ }^{1}$, MORIO TONOGI ${ }^{1}$, AI KOTANI $^{6}$ and KENICHI IMAI ${ }^{2}$ \\ ${ }^{I}$ Department of Oral and Maxillofacial Surgery, Nihon University School of Dentistry, Tokyo, Japan; \\ ${ }^{2}$ Department of Microbiology, Nihon University School of Dentistry, Tokyo, Japan; \\ ${ }^{3}$ Department of Periodontology, Nihon University School of Dentistry at Matsudo, Chiba, Japan; \\ ${ }^{4}$ Department of Endodontics, Nihon University School of Dentistry, Tokyo, Japan; \\ ${ }^{5}$ Division of Hematology and Rheumatology, Department of Medicine, \\ Nihon University School of Medicine, Tokyo, Japan; \\ ${ }^{6}$ Department of Hematological Malignancy, Institute of Medical Science, Tokai University, Kanagawa, Japan
}

\begin{abstract}
Background/Aim: Epstein-Barr virus (EBV) associates with human chronic periodontitis (CP) progression. We previously demonstrated that butyric acid $(B A)$, produced by periodontopathic bacteria, induced EBV lytic switch activator BZLF1 expression. We investigated whether short chain fatty acids (SCFAs) in CP patients' saliva enabled EBV reactivation. Materials and Methods: Saliva was collected from seven CP patients and five periodontally healthy individuals. SCFAs were quantified using HPLC. BZLF1 mRNA and its pertinent protein ZEBRA were determined with Real-time PCR and western blotting. Histone $\mathrm{H} 3$ acetylation ( $\mathrm{AcH} 3$ ) was further examined. Results: BZLF1 mRNA expression and transcriptional activity in EBV-infected Daudi cells were induced only when treated with the CP saliva. Among SCFAs, BA alone correlated significantly with the BZLF1 transcription $(r=0.88 ; p<0.02)$. As expected, $C P$ patients' saliva induced AcH3. Conclusion: $B A$ in saliva may play a role in $E B V$ reactivation and hence contribute to $E B V$ related disease progression in $\mathrm{CP}$ patients.
\end{abstract}

This article is freely accessible online.

Correspondence to: Kenichi Imai, DDS, Ph.D., Department of Microbiology, Nihon University School of Dentistry, 1-813 Kanda-Surugadai, Chiyoda-ku, Tokyo 101-8310, Japan. Tel: +81 332198115, Fax: +81 332198317, e-mail: imai.kenichi@nihon-u.ac.jp

Key Words: Epstein-Barr virus, periodontitis, butyric acid, saliva, reactivation.
Chronic periodontitis (CP) is a complex chronic inflammatory microbial disease that is prevalent in humans worldwide $(1,2)$. Severe CP can result in the loosening of teeth, occasional pain, periodontal bone loss, and eventual tooth loss $(1,2)$. Mounting evidence has indicated that $\mathrm{CP}$ is a risk factor for aspiration pneumonia, chronic obstructive pulmonary disease, diabetes, and pre-term birth $(1,2)$. Although no single etiological agent has been identified, a number of putative bacteria, such as Porphyromonas gingivalis and Fusobacterium nucleatum, are considered to be associated with $\mathrm{CP}$ and, thus, are used as diagnostic markers $(2,3)$. Recently, members of the herpes virus family, such as Epstein-Barr virus (EBV), have been suggested to be involved in the aetiology of CP because bacterial activity alone does not adequately explain the clinical characteristics of CP (4-8).

Similar to other herpes viruses, EBV establishes a persistent infection in the human host, and its life cycle has both lytic and latent phases $(9,10)$. The EBV-encoded immediate-early BZLF1 gene encodes ZEBRA, a sequence-specific DNAbinding protein that is a member of the bZIP family of leucine-zipper transcriptional activators $(9,10)$. Since ZEBRA can transactivate early and late genes of EBV, and thereby induce the lytic cycle, this viral transcriptional activator is a master regulator of the transition from latency to the lytic replication cycle $(9,10)$. EBV is frequently reactivated in immunocompromised hosts and can induce infectious mononucleosis, as well as several malignancies, such as Burkitt lymphoma and nasopharyngeal carcinoma (9-12).

Many studies have demonstrated that the amount of EBV DNA detected in the periodontal pockets and gingival tissues of $\mathrm{CP}$ patients is correlated with disease severity (4-8). Accordingly, we previously reported that EBV DNA was more 
frequently detected in deep, rather than shallow, periodontal pockets among Japanese patients with $\mathrm{CP}$ and healthy controls $(13,14)$. We also observed a large number of EBV-encoded small RNA-positive B-cells in the gingival tissues of CP patients (13). Although EBV is epidemiologically involved in the aetiology of $\mathrm{CP}$, the process by which latent EBV is reactivated in the oral cavity remains unclear.

EBV is usually transmitted through saliva and replicates in the salivary glands, oral mucosal membrane, nasopharyngeal epithelium, and B cells $(6,7,11,12,15,16)$. In addition, the saliva of CP patients contains EBV-infected B cells, higher levels of EBV DNA, and greater concentrations of periodontopathic bacteria $(6,7,15-17)$, suggesting a relationship between microbial interactions and the aetiology of CP. We have also reported that although short-chain fatty acids (SCFAs) are secreted extracellularly by $P$. gingivalis and $F$. nucleatum, only butyric acid (BA) can induce reactivation of EBV (18). These observations suggest that EBV reactivation may be caused by BA in the saliva of CP patients. However, no studies have yet to evaluate the amount of $\mathrm{BA}$ in the saliva of $\mathrm{CP}$ patients and determine whether the saliva can reactivate EBV.

Therefore, the aim of the present study was to assess the levels of BA in the saliva of CP patients, which could efficiently induce the expression of the EBV lytic switch activator $B Z L F 1$, and to determine for the first time whether there is a possible pathophysiological link with EBV reactivation. In addition, this article discusses how this relationship may pertain to the aetiology of $\mathrm{CP}$.

\section{Materials and Methods}

Study approval and participants. The study protocol was approved by the Institutional Internal Review and Ethics Board of the Nihon University School of Dentistry (Tokyo, Japan; approval no.: EP17D006) and conducted in accordance with the tenets of the Declaration of Helsinki. The study cohort included seven CP patients (mean age: $53.1 \pm 13.7$ years) and five periodontal healthy individuals (mean age: $32.6 \pm 6.1$ years). Written informed consent was obtained from each study participant after all procedures had been fully explained.

Reagents. BA was purchased from Wako Pure Chemical Industries, Ltd. (Osaka, Japan). Antibodies (Abs) against ZEBRA and $\beta$-actin were purchased from Santa Cruz Biotechnology, Inc., Dallas, TX, USA), whereas those against acetylated histone 3 were obtained from Thermo Fisher Scientific (Waltham, MA, USA) and those against non-acetylated histone 3 were purchased from Abcam (Cambridge, UK).

Saliva collection. All of the study participants received dental care at Nihon University School of Dentistry. Periodontal status was assessed based on the probing pocket depth (PPD), clinical attachment level (CAL), and bleeding on probing (BOP). The PPD and CAL were measured using a PCP11 probe (Hu-Friedy Mfg. Co., LLC, Chicago, IL, USA). CP was defined as the presence of at least two sites with a PPD of $\geq 5 \mathrm{~mm}$ and attachment loss of $>6 \mathrm{~mm}$.
The healthy controls had no clinical signs of gingivitis, attachment loss, or detectable bone loss on radiographic examinations, and PPD was $\leq 3 \mathrm{~mm}$. All patients were systemically healthy with no history of periodontal treatment or any type of antibiotic therapy for at least 3 months prior to participation in this study. About $10 \mathrm{ml}$ of saliva were collected from each participant. After centrifugation to remove cells and debris, the supernatant of the collected saliva was sterilised by passing through a $0.22-\mu \mathrm{m}$ pore filter membrane and then either immediately analysed or stored at $-80^{\circ} \mathrm{C}$ for future use.

Cell culture. Daudi cells, which are well-characterised EBV-positive human Burkitt lymphoma-derived cells, and B95-8-221 Luc cells $(18,19)$, which were stably transfected with the $B Z L F 1$ promoter, were maintained at $37^{\circ} \mathrm{C}$ in Roswell Park Memorial Institute 1640 medium (Sigma-Aldrich Corporation, St. Louis, MO, USA) containing $10 \%$ heat-inactivated fetal bovine serum (Sigma-Aldrich Corporation), penicillin $(100 \mathrm{U} / \mathrm{ml})$, and streptomycin $(100 \mathrm{mg} / \mathrm{ml})$. For the stimulation experiments, cells $\left(1.0 \times 10^{6}\right.$ cells $/ 1.0-\mathrm{ml}$ well $)$ were treated with saliva or BA.

Quantification of SCFAs. SCFAs [BA, propionic acid (PA), acetic acid (AA), isoBA, and isovaleric acid] were quantified using ion exclusion high-performance liquid chromatography (HPLC), as described previously $(18,20)$. Briefly, each saliva sample was mixed with $12 \%$ perchloric acid, filtered through a cellulose acetate membrane filter (Cosmonice Filter W, pore size: $0.45 \mu \mathrm{m}$; Nacalai Tesque, Inc., Kyoto, Japan), and then injected into a SIL-10 auto injector (Shimadzu Corporation, Kyoto, Japan). SCFAs were separated using a serial organic acid column and a guard column with isocratic elution of p-toluene sulfonic acid aqueous solution and detected using an electronic conductivity detector.

Preparation of $m R N A$ and real-time polymerase chain reaction $(P C R)$. The experimental procedures for RNA purification and realtime PCR were performed as previously described $(18,21)$. Briefly, Daudi cells were washed once with ice-cold phosphate-buffered saline (PBS) and homogenized using a QIAshredder (QIAGEN, Alameda, CA, USA), while total RNA was purified using a RNeasy Mini Kit (QIAGEN). For cDNA synthesis, total RNA (1 $\mu \mathrm{g})$ was reverse transcribed using an RNA PCR kit (PrimeScript; Takara Bio, Shiga, Japan). The resulting cDNA mixture was subjected to real-time PCR analysis using SYBR Premix Ex Taq solution (Takara Bio) containing $5 \mu \mathrm{M}$ sense and antisense primers. The primer sequences used for the amplification of each gene were as follows: BZLF1 forward (5-TTC CAC AGC CTG CAC CAG TG-3) and reverse (5- GGC AGC AGC CAC CTC ACG GT -3); and glyceraldehyde-3-phosphate dehydrogenase (GAPDH), forward (5-ACC AGC CCC AGC AAG AGC ACA AG-3) and reverse (5-TTC AAG GGG TCT ACA TGG CAA CTG-3). PCR assays were performed using a TP-800 Thermal Cycler Dice Real-Time System (Takara Bio) and analyzed using the software provided by the device manufacturer. The thermal cycling conditions were 40 cycles at $95^{\circ} \mathrm{C}$ for $5 \mathrm{~s}, 60^{\circ} \mathrm{C}$ for $30 \mathrm{~s}$, and $72^{\circ} \mathrm{C}$ for $1 \mathrm{~min}$. All real-time PCR experiments were performed in triplicates, and the specificity of each product was verified via a melting curve analysis. The calculated gene expression levels were normalized to $G A P D H$ mRNA levels.

Luciferase assay. Luciferase assay was then performed using a DualLuciferase Reporter Assay System (Promega), according to the 
manufacturer's instructions. The experimental procedure for the luciferase assay has been previously reported $(20,21)$. B95-8-221 Luc. cells were harvested and the extracts were subjected to luciferase assay using the Dual-Luciferase Assay System ${ }^{\mathrm{TM}}$ (Promega). All the experiments were carried out in triplicates and the data are presented as the fold increase in luciferase activities (means \pm S.D.) relative to the control of three independent transfections.

Immunoblot assay. The experimental procedures for immunoprecipitation and immunoblotting were performed according to previously published protocols $(22,23)$. Briefly, cells were harvested with lysis buffer [25 mM HEPES-NaOH ( $\mathrm{pH} 7.9), 150$ $\mathrm{mM} \mathrm{NaCl}, 1.5 \mathrm{mM} \mathrm{MgCl} 2,0.2 \mathrm{mM}$ EDTA, $0.3 \% \mathrm{NP}-40,1 \mathrm{mM}$ dithiothreitol, $0.5 \mathrm{mM}$ phenylmethylsulfonyl fluoride], the proteins were separated by SDS-PAGE and transferred to a polyvinylidene fluoride membrane (EMD Millipore Corporation, Billerica, MA, USA). The protein content was measured by a detergent-compatible protein assay kit (Bio-Rad, Hercules, Hercules, CA, USA). All membranes were treated with ECL prime detection reagent (Thermo Fisher Scientific) prior to examination. All bands were visualized using a ChemiDoc XRS System (Bio-Rad).

Statistical analysis. Comparison of two groups was performed using the two-tailed Student's $t$-test. The correlation coefficient $(\mathrm{r})$ was calculated where applicable. A probability $(p)$ value of $<0.05$ was considered statistically significant.

\section{Results}

Saliva of CP patients contains relatively high levels of SCFAs. Previous studies have reported that the periodontal pockets and dental plaques of CP patients contain high concentrations (mM levels) of SCFAs (24-26). However, the amounts of SCFAs have not been investigated in the saliva of Japanese CP patients. Therefore, we measured the concentrations of SCFAs in the saliva of seven CP patients and five healthy controls by HPLC. As presented in Figure 1 , the saliva of $\mathrm{CP}$ patients contained significantly higher levels $(p<0.01)$ of BA, PA, and AA. On the other hand, the amounts of isoBA and isovaleric acid in the saliva were very low. The concentrations of BA, PA, and AA in the saliva of CP patients were 0.31-1.37, 0.49-1.35, and 2.12-5.81 mM, respectively. On average, the saliva of $\mathrm{CP}$ patients contained about 33.3-, 3.3-, and 2.4-fold higher levels of BA, PA, and AA than that of the healthy controls.

Saliva of CP patients induces expression of BZLF1. Since high concentrations of SCFAs were found in the saliva of $\mathrm{CP}$ patients, we investigated whether the saliva can reactivate EBV. Real time-PCR was conducted to evaluate the effect of the saliva of seven CP patients and five healthy controls at a 1:2 dilution on BZLF1 mRNA expression in Daudi cells. As presented in Figure 2A, mRNA levels of the EBV lytic gene $B Z L F 1$ were significantly higher in cells treated with the saliva of CP patients than of that of the healthy controls. Interestingly, there was a significant correlation between BA concentrations and BZLF1 transcript levels ( $\mathrm{r}=0.88 ; p<0.02)$. The concentration of BA in the saliva of CP patients $(0.31$ $1.37 \mathrm{mM}$ ) induced BZLF1 expression in a concentrationdependent manner (Figure 2C). In contrast, no such effect was observed with PA and AA (data not shown). Next, we examined the effect of saliva on gene expression of the $B Z L F 1$ promoter using the luciferase assay. As demonstrated in the results presented in Figure 2D, the saliva of $C P$ patients transactivated the BZLF1 promoter in B95-8-221 Luc cells.

Saliva of CP patients induces expression of ZEBRA. Next, we examined the expression of the lytic switch transactivator ZEBRA by exposure to the saliva of CP patients. As presented in Figure 3, the saliva of the healthy controls had no effect on the expression of ZEBRA in Daudi cells. However, the addition of the saliva of $\mathrm{CP}$ patients increased the expression of ZEBRA.

Hyperacetylation of histones by the saliva of CP patients. BA is known to inhibit the enzymatic activity of histone deacetylase (HDAC) by competing with the HDAC substrate for the enzyme's active site pocket, which contains the catalytic center (27), thus stimulating transcription of various genes, including $B Z L F 1(9,10,28)$. We have previously demonstrated that the culture supernatant from periodontopathic bacteria, which contains high concentrations of BA, can inhibit HDACs, thereby increasing the level of histone acetylation and the transcriptional activity of the BZLF1 gene (18). Next, we examined the effects of saliva and BA on histone acetylation by western blotting with Abs specific for acetylated histone $\mathrm{H} 3$. As presented in Figure 4, although there was no effect by the saliva of the healthy controls, both the saliva of CP patients and BA induced acetylation of histone H3 (Figure 4A, B). In contrast, no such effect was observed with the other tested SCFAs (data not shown).

\section{Discussion}

Reactivation of latent EBV is associated with progeny virus production and several human diseases (9-12). Therefore, elucidation of the mechanisms that promote or disrupt EBV latency in infected individuals is required to understand the pathobiology of EBV infection and to develop preventive measures and novel therapies. However, the trigger that is responsible for the switch from latency to the lytic cycle in individuals latently infected with EBV remains unclear. In this study, we examined the biological actions of the saliva of CP patients and healthy controls on the reactivation of EBV infection.

It has been reported that more than sufficient concentrations of BA are present in the dental plaques (range $=4.7-13.8 \mathrm{mM}$ ) $(25,26)$ and periodontal pockets $($ mean $=2.6 \pm 0.4 \mathrm{mM})$ of patients with periodontal disease (24), whereas the BA concentration is 


\section{Butyric acid}

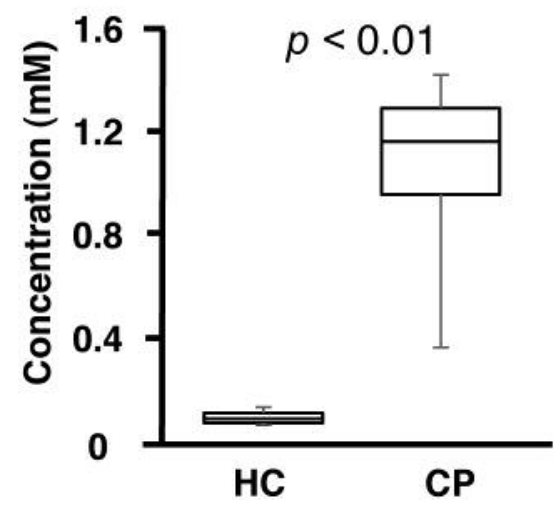

Acetic acid

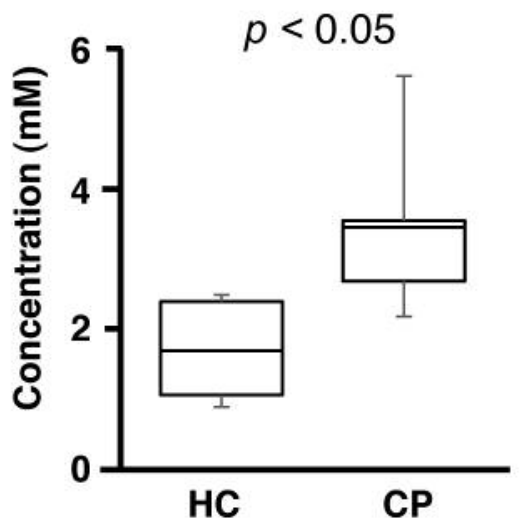

Propionic acid

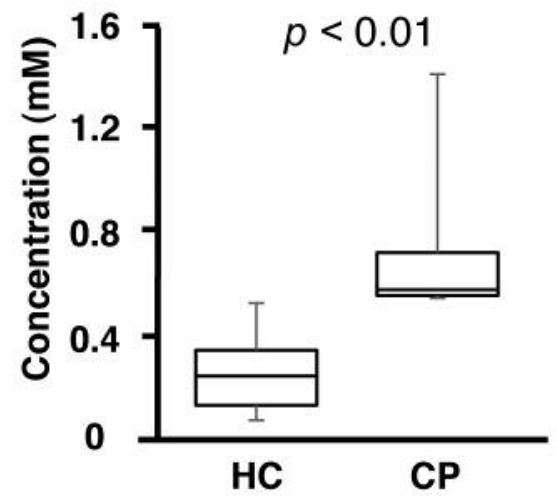

Isobutyric acid

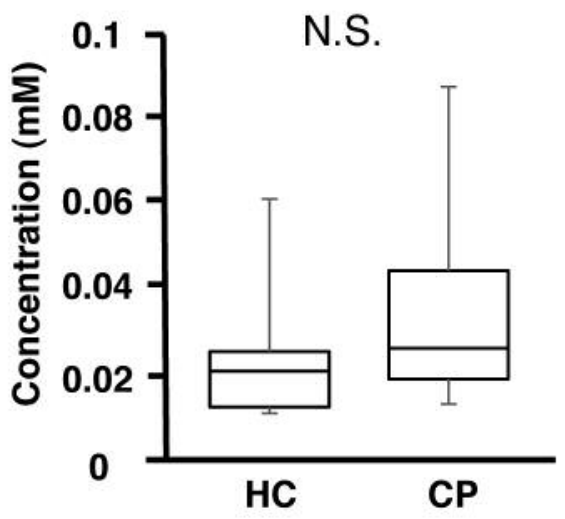

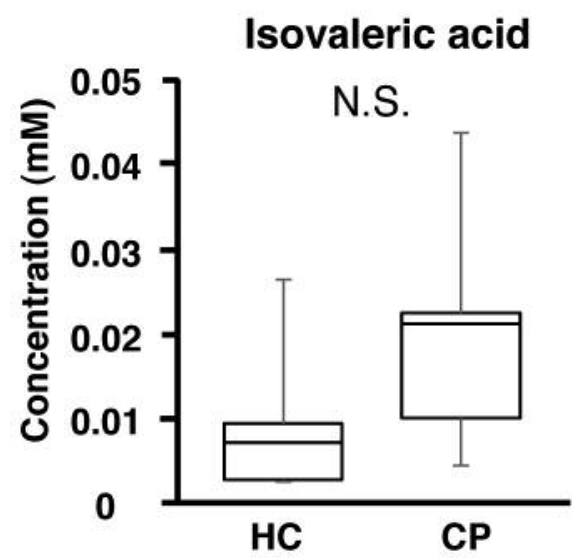

Figure 1. Saliva of CP patients contains higher levels of SCFAs. Levels of of butyric acid, propionic acid, acetic acid, isobutyric acid, and isovaleric acid in the saliva of seven CP patients and five healthy controls. HC: Healthy control; CP: chronic periodontitis; N.S.: not significant.

below the detection limits in healthy sites, suggesting that BA may play a role in the initiation of EBV reactivation and contribute to the clinical progression of EBV-related diseases. The results of the present study revealed significantly higher levels of BA in the saliva of CP patients, which could efficiently induce BZLF1 transcription. These observations suggest that the BA content in the saliva of CP patients might be involved in the progression of EBV-related diseases as well as periodontitis. 

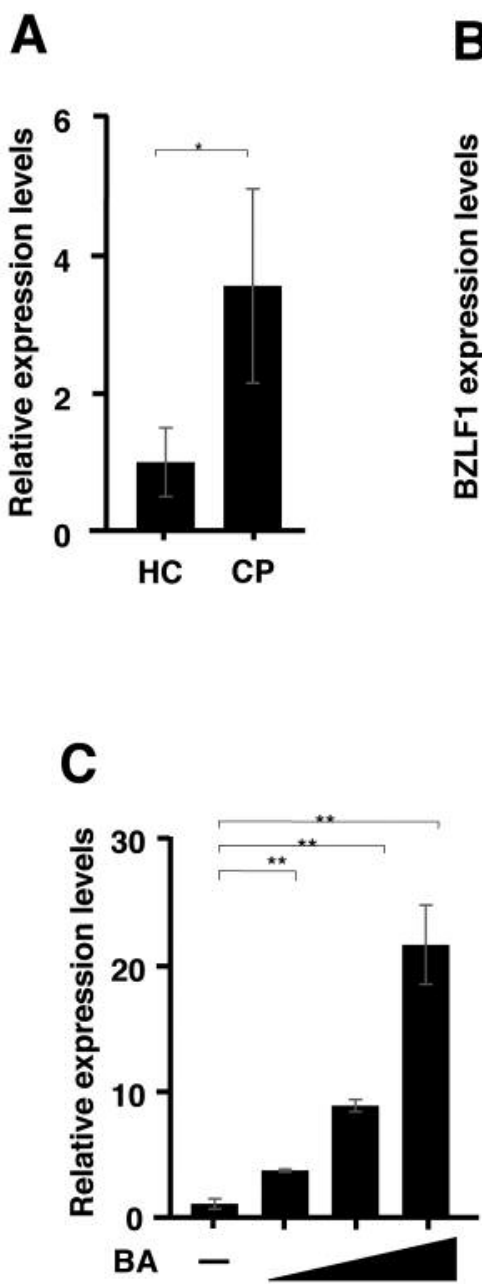
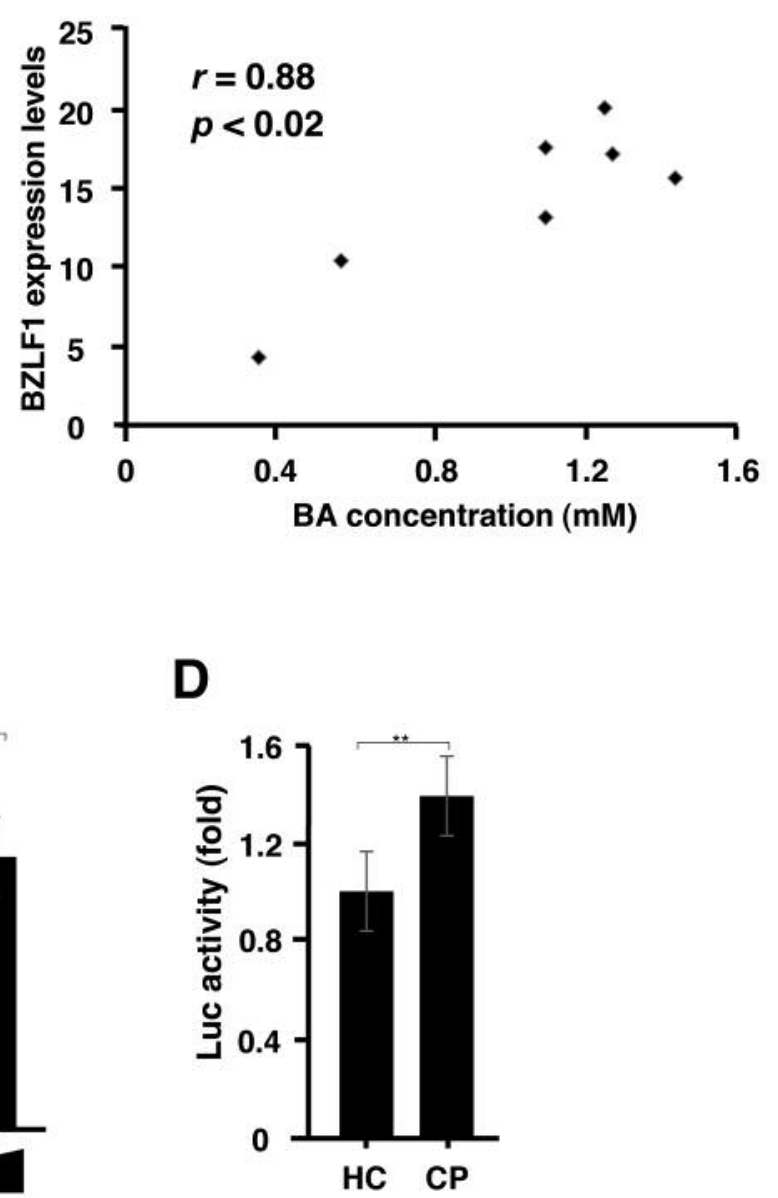

Figure 2. Induction of BZLF1 gene expression by the saliva of CP patients. (A) Daudi cells were incubated with the saliva of seven CP patients and five healthy controls at a 1:2 dilution (saliva volume vs. total cell culture medium volume) for $24 \mathrm{~h}$. Real-time PCR analysis was conducted with specific primers to detect BZLF1 mRNA expression. (B) The correlation coefficient $(r)$ was calculated between butyric acid (BA) concentrations and BZLF1 mRNA levels. (C) Daudi cells were treated with BA $(0.5,1.0$, or $1.5 \mathrm{mM})$ for $24 \mathrm{~h}$, and BZLF1 $\mathrm{mRNA}$ expression was assessed. (D) B95-8-221 Luc cells were treated with the saliva of seven CP patients and five healthy controls at a 1:2 dilution for $48 \mathrm{~h}$. The luciferase activity of each cell lysate was then measured. The values are presented as mean \pm standard deviation $(n=3) . * * p<0.01, * p<0.05$.

Since BZLF1 expression is a key step in the reactivation from latency in EBV-infected cells $(9,10)$, we focused on the expression of this transcriptional activator. Although, neither BZLF1 mRNA nor ZEBRA protein was detectable during latency, high levels of anti-BZLF1 Abs in blood are associated with an increased EBV serum load in EBVinfected patients (29). We observed that high concentrations (mM levels) of BA, AA, and PA are present in the saliva of Japanese CP patients (Figure 1). Interestingly, there was a significant correlation between $\mathrm{BA}$ concentrations and the levels of $B Z L F 1$ transcripts ( $\mathrm{r}=0.88 ; p<0.02$; Figure $2 \mathrm{~B})$. In fact, BA was the only acid in the saliva of $\mathrm{CP}$ patients that induced BZLF1 expression (Figure 2C). These results support the findings of a previous in vitro study, which reported that among several SCFAs in the culture supernatant of periodontopathic bacteria, only butyrate reactivated EBV, whereas non-butyrate-producing bacteria did not (18).

Recent studies have revealed a new mechanism that regulates the maintenance and reversal of EBV latency, which involves nucleosome configurations and histone modifications. In the latent state, the $B Z L F 1$ gene promoter is bound by histone proteins into a chromatin structure that serves to repress the transcription of $\operatorname{BZLF1}(9,10)$. Hyperacetylation of core histone proteins adjacent to the $B Z L F 1$ promoter was correlated with transcriptional activation of BZLF1, whereas hypoacetylation mediated by 


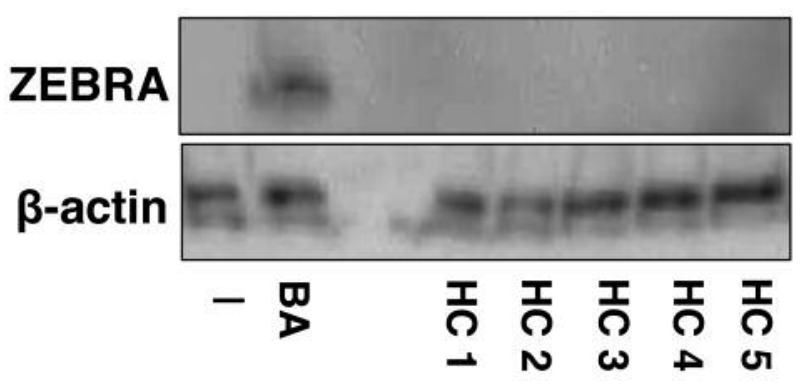

ZEBRA

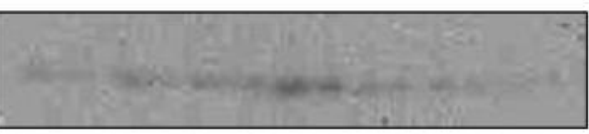

$\beta$-actin

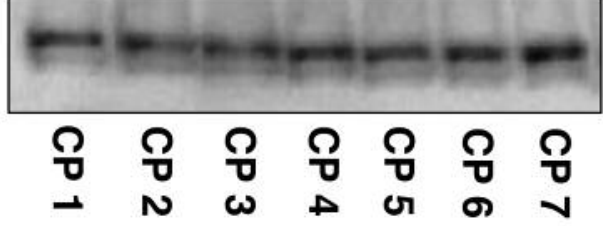

Figure 3. Saliva of CP patients induced ZEBRA expression. Daudi cells were treated with the saliva of seven $C P$ patients and five healthy controls at a 1:2 dilution or $B A(2 \mathrm{mM}$, positive control) for $36 \mathrm{~h}$. The lysates were harvested, and ZEBRA levels were assessed by immunoblotting using specific Abs. $\beta$-actin was used as an internal control. BA: Butyric acid.

HDAC was correlated with its repression, which is considered responsible for the maintenance of latency (9, 10). Since BA is one of the most potent inhibitors of HDACs and our previous report indicated that BA in the culture supernatant of periodontopathic bacteria promotes histone acetylation and the transcriptional activity of the BZLF1 gene (18), we examined whether the saliva of CP patients induced histone acetylation. We found that the saliva of $\mathrm{CP}$ patients induced Lys acetylation of histone H3 in EBV-infected cells (Figure 4). Our previous study indicated that no such activity occurred with $P$. gingivalis or bacterial components, such as lipopolysaccharide and fimbriae (16). Although it is necessary to assess other factors contained in saliva, such as cytokines and enzymes, our findings suggested that $\mathrm{H} 3$ histone acetylation and BZLF1 expression are ascribable to BA contained in the saliva of CP patients.

The saliva of patients with periodontitis contains EBVinfected B cells, and bleeding of the gums is often observed in these patients $(7,15-17)$. In addition, it was recently reported that EBV infects the oral epithelial cells of patients with periodontitis in addition to the epithelial cells of the upper aerodigestive tract (30). The extent of gingival epithelial EBV infection is correlated with the severity of CP (30). Moreover, previous reports, as well as the present study, indicated that
A
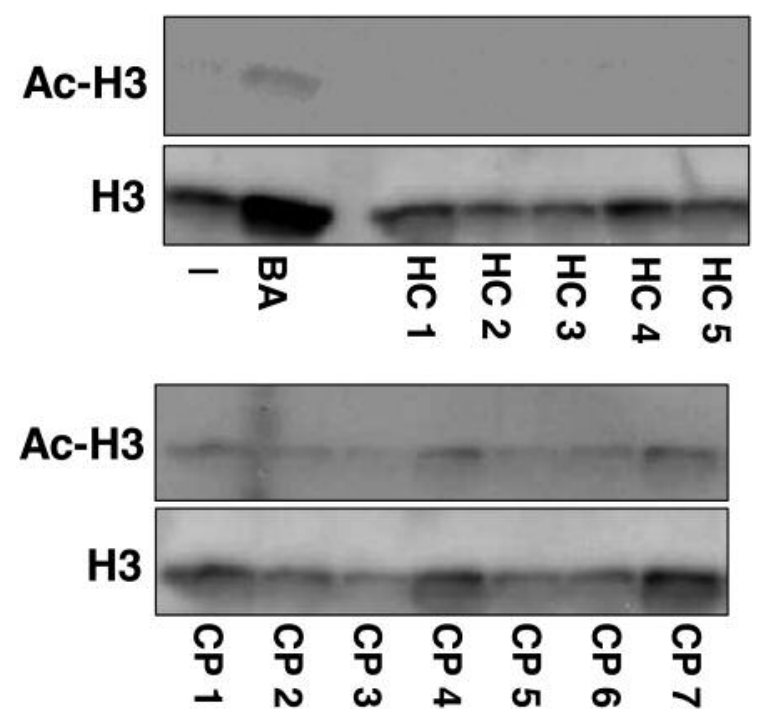

B

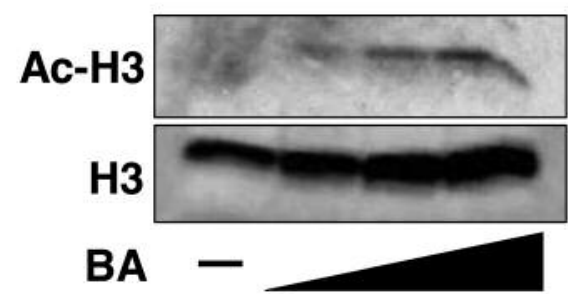

Figure 4. Hyperacetylation of histones by the saliva of CP patients. (A) Daudi cells were treated with the saliva of seven $C P$ patients and five healthy controls at a 1:2 dilution or BA (2 $\mathrm{mM})$ for $36 \mathrm{~h}$. (B) Daudi cells were treated with $B A(0.5,1.0$, or $1.5 \mathrm{mM})$ for $36 \mathrm{~h}$. The lysates were harvested, and acetylated histone proteins were assessed by western blot analysis using Abs against acetylated histone 3. The unmodified H3 protein was used as control. BA: Butyric acid.

EBV also contributes to the progression of periapical periodontitis $(20,31)$. These findings and previous observations suggest the potential risks of BA in saliva for the progression of periodontitis and periapical periodontitis. We assume that microbial synergy by the interaction between periodontopathic bacteria and EBV leads to the following negative chain of pathological events in the oral cavity: 1) periodontopathic anaerobic bacteria, such as $P$. gingivalis and $F$. nucleatum, produce $\mathrm{BA}$; 2) BA induces EBV reactivation; 3) EBV impairs local host defences, 4) which leads to increased proliferation of periodontopathic bacteria; 5) increased BA and inflammatory cytokine production by the synergistic effects of EBV and periodontopathic bacteria; and 6) periodontitis escalation. 
Periodontitis and EBV are spreading worldwide. Although our findings suggest a relationship between the saliva of patients with periodontitis and EBV reactivation, additional basic and clinical studies with greater numbers of cases are needed. Furthermore, prevention and early treatment of periodontitis involving elimination of BA-producing bacteria could effectively block further clinical progression of EBV infection.

\section{Conflicts of Interest}

The Authors have no conflicts of interest to declare in regard to this study.

\section{Authors' Contributions}

R.K., A.K., and K.I. conceived and designed the experiments. R.K., K.N., and N.W. performed the experiments, analysed the data, contributed reagents/materials/analytical tools, prepared the figures, and reviewed drafts of the article. Y.O., O.T., and M.T. contributed reagents/materials/analytical tools, analysed the data, and reviewed drafts of the paper. T.K. and M.T. contributed to the discussion, analysed the data, and reviewed the drafts of the article. K.I. performed the experiments, analysed the data, authored or reviewed drafts of the article.

\section{Acknowledgements}

This work was supported by JSPS KAKENHI (grant no. 16K11526), the Uemura Fund (Dental Research Center, Nihon University School of Dentistry), and a Nihon University Multidisciplinary Research Grant for 2017.

\section{References}

1 Hajishengallis G: Periodontitis: From microbial immune subversion to systemic inflammation. Nat Rev Immunol 15(1): 30-44, 2015. PMID: 25534621. DOI: $10.1038 /$ nri3785

2 Darveau RP: Periodontitis: A polymicrobial disruption of host homeostasis. Nat Rev Microbiol 8(7): 481-490, 2010. PMID: 20514045. DOI: $10.1038 /$ nrmicro 2337

3 Holt SC and Ebersole JL: Porphyromonas gingivalis, Treponema denticola, and Tannerella forsythia: The "red complex", a prototype polybacterial pathogenic consortium in periodontitis. Periodontol 2000 38(1): 72-122, 2005. DOI: 10.1111/j.16000757.2005.00113.x

4 Contreras A, Nowzari H and Slots J: Herpesviruses in periodontal pocket and gingival tissue specimens. Oral Microbiol Immunol 15(1): 15-18, 2000. PMID: 11155159. DOI: 10.1034/j.1399302x.2000.150103.x

$5 \mathrm{Lu} \mathrm{H}$, Zhu C, Li F, Xu W, Tao D and Feng X: Putative periodontopathic bacteria and herpesviruses in pregnant women: a case-control study. Sci Rep 6: 27796, 2016. PMID: 27301874. DOI: $10.1038 /$ srep2779

6 Slots J, Saygun I, Sabeti M and Kubar A: Epstein-Barr virus in oral diseases. J Periodontal Res 41(4): 235-244, 2006. PMID: 16827715. DOI: 10.1111/j.1600-0765.2006.00865.x

7 Saygun I, Kubar A, Özdemir A and Slots J: Periodontitis lesions are a source of salivary cytomegalovirus and Epstein-Barr virus.
J Periodontal Res 4O(2): 187-191, 2005. PMID: 15733155. DOI: 10.1111/j.1600-0765.2005.00790.x

8 Konstantinidis A, Sakellari D, Papa A and Antoniadis A: Realtime polymerase chain reaction quantification of Epstein-Barr virus in chronic periodontitis patients. J Periodontal Res 40(4): 294-298, 2005. PMID: 15966906. DOI: 10.1111/j.16000765.2005.00796.x

9 Jenkins PJ, Binne UK and Farrell PJ: Histone acetylation and reactivation of Epstein-Barr virus from latenc. J Virol 74(2): 710-720, 2000. PMID: 10623733. DOI:10.1128/jvi.74.2.710720.2000

10 Bryant $\mathrm{H}$ and Farrell PJ: Signal Transduction and transcription factor modification during reactivation of Epstein-Barr Virus from latency. J Virol 76(20): 10290-10298, 2002. PMID: 12239305. DOI: $10.1128 /$ jvi.76.20.10290-10298.2002

11 Schmidt CW and Misko IS: The ecology and pathology of Epstein-Barr virus. Immunol Cell Biol 73: 489-504, 1995. PMID: 8713470. DOI: 10.1038/icb.1995.79

12 Thorley-Lawson DA and Gross A: Persistence of the EpsteinBarr virus and the origins of associated lymphomas. N Engl $\mathbf{J}$ Med 350(13): 1328-1337, 2004. PMID: 15044644. DOI: 10.1056/NEJMra032015

13 Kato A, Imai K, Ochiai K and Ogata Y: Higher prevalence of Epstein-Barr virus DNA in deeper periodontal pockets of chronic periodontitis in Japanese patients. PLoS One 8(8): e71990, 2013. PMID: 23991022. DOI: 10.1371/journal.pone.0071990

14 Kato A, Imai $\mathrm{K}$, Ochiai $\mathrm{K}$ and Ogata $\mathrm{Y}$ : Prevalence and quantitative analysis of Epstein-Barr virus DNA and Porphyromonas gingivalis associated with Japanese chronic periodontitis patients. Clin Oral Investig 19(7): 1605-1610, 2015. PMID: 29233156. DOI: 10.1186/s12903-017-0438-6

15 Slots J: Herpesviral-bacterial synergy in the pathogenesis of human periodontitis. Curr Opin Infect Dis 20(3): 278-283, 2007. PMID: 17471038. DOI: 10.1097/QCO.0b013e3280964da0

16 Contreras A, Zadeh HH, Nowzari H and Slots J: Herpesvirus infection of inflammatory cells in human periodontitis. Oral Microbiol Immunol 14(4): 206-212, 1999. PMID: 10551163. DOI: $10.1034 /$ j.1399-302x.1999.140402.x

17 Chalabi M, Rezaie F, Moghim S, Mogharehabed A, Rezaei M and Mehraban B: Periodontopathic bacteria and herpesviruses in chronic periodontitis. Mol Oral Microbiol 25(3): 236-240, 2010. PMID: 20536751. DOI: 10.1111/j.2041-1014.2010.00571.x

18 Imai K, Inoue H, Tamura M, Cueno ME, Inoue H, Takeichi O, Kusama K, Saito I and Ochiai K: The periodontal pathogen Porphyromonas gingivalis induces the Epstein-Barr virus lytic switch transactivator ZEBRA by histone modification. Biochimie 94(3): 839-846, 2012. PMID: 22178321. DOI: 10.1016/j.biochi.2011.12.001

19 Inoue H, Mishima K, Yamamoto-Yoshida S, Ushikoshi-Nakayama R, Nakagawa Y, Yamamoto K, Ryo K, Ide F and Saito I: Aryl hydrocarbon receptor-mediated induction of EBV reactivation as a risk factor for Sjögren's syndrome. J Immunol 188(9): 46544662, 2012. PMID: 22467650. DOI: 10.4049/jimmunol.1101575

20 Makino K, Takeichi O, Imai K, Inoue H, Hatori K, Himi K, Saito I, Ochiai K and Ogiso B: Porphyromonas endodontalis reactivates latent Epstein-Barr virus. Int Endod J 51(12): 14101419, 2018. PMID: 29858508. DOI: 10.1111/iej.12959

21 Imai K, Kamio N, Cueno ME, Saito Y, Inoue H, Saito I and Ochiai K: Role of the histone H3 lysine 9 methyltransferase Suv39 h1 in maintaining Epstein-Barr virus latency in B95-8 
cells. FEBS J 281(9): 2148-2158, 2014. PMID: 24588869. DOI $10.1111 /$ febs. 12768

22 Imai $\mathrm{K}$, Togami $\mathrm{H}$ and Okamoto $\mathrm{T}$ : Involvement of histone $\mathrm{H} 3$ lysine 9 (H3K9) methyltransferase G9a in the maintenance of HIV-1 latency and its reactivation by BIX01294. J Biol Chem 285(22): 16538-16545, 2010. PMID: 20335163. DOI: 10.1074/ jbc.M110.103531

23 Watanabe N, Nodomi K, Koike R, Kato A, Takeichi O, Kotani AI, Kaneko T, Sakagami H, Takei M, Ogata Y, Sato S and Imai $\mathrm{K}$ : EBV LMP1 in gingival epithelium potentially contributes to human chronic periodontitis via inducible IL8 production. in vivo 33(6): 1793-1800, 2019. PMID: 31662504. DOI: 10.21873/ invivo. 11670

24 Niederman R, Buyle-Bodin Y, Lu BY, Robinson P and Naleway C: Short-chain carboxylic acid concentration in human gingival crevicular fluid. J Dent Res 76(1): 575-579, 1997. PMID: 9042080. DOI: 10.1177/00220345970760010801

25 Margolis HC, Duckworth JH and Moreno EC: Composition and buffer capacity of pooled starved plaque fluid from caries-free and caries-susceptible individuals. J Dent Res 67(12): 1476-1482, 1988. PMID: 3198845. DOI: 10.1177/00220345880670120701

26 Moreno EC and Margolis HC: Composition of human plaque fluid. J Dent Res 67(11): 1181-1189, 1988. PMID: 3045178. DOI: $10.1177 / 00220345880670090701$

27 Riggs MG, Whittaker RG, Neumann JR and Ingram VM: nButyrate causes histone modification in HeLa and Friend erythroleukaemia cells. Nature 268(5619): 462-464, 1977. PMID: 268489. DOI: $10.1038 / 268462 \mathrm{a} 0$
28 Saemundsen AK, Kallin B and Klein G: Effect of n-butyrate on cellular and viral DNA synthesis in cells latently infected with Epstein-Barr virus. Virology 107(2): 557-561, 1980. PMID: 6256952. DOI: 10.1016/0042-6822(80)90326-8

29 Drouet E Brousset P, Fares F, Icart J, Verniol C, Meggetto F, Schlaifer D, Desmorat-Coat H, Rigal-Huguet F, Niveleau A and Delsol G: High Epstein-Barr virus serum load and elevated titers of anti-ZEBRA antibodies in patients with EBV-harboring tumor cells of Hodgkin's disease. J Med Virol 57(4): 383-389, 1999. PMID: 10089051. DOI: 10.1002/(sici)1096-9071(199904)57: 4<383::aid-jmv10>3.0.co;2-3

30 Vincent-Bugnas S, Vitale S, Mouline CC, Khaali W, Charbit Y, Mahler P, Prêcheur I, Hofman P, Maryanski JL and Doglio A: EBV infection is common in gingival epithelial cells of the periodontium and worsens during chronic periodontitis. PLoS One 8(2): e80336, 2013. PMID: 24367478. DOI: 10.1371/ journal.pone. 0080336

31 Makino K, Takeichi O, Hatori K, Imai K, Ochiai K and Ogiso B: Epstein-Barr virus infection in chronically inflamed periapical granulomas. PLoS One 10(4): e0121548, 2016. PMID: 25884725. DOI: 10.1371/journal.pone.0121548

Received November 7, 2019

Revised November 26, 2019

Accepted November 29, 2019 